\title{
Research of Sensors Failures Diagnosis Based on Noise Analysis Aidong $\mathrm{Xu}^{1}{ }^{1}$,Yuanfeng Liu1 ${ }^{1,2}$, Jing Jiang ${ }^{3}$,Mingzhe Liu ${ }^{1}$,Bingjun Yan ${ }^{1}$ \&Qinning Liu ${ }^{1,2}$ \\ ${ }^{1}$ Shenyang Institute of Automation, Chinese Academy of Sciences, Shenyang, 110016 \\ ${ }^{2}$ University of Chinese Academy of Sciences, Beijing, 100049 \\ ${ }^{3}$ University of Western Ontario, Canada
}

Keywords: noise analysis, diagnosis, sensor failure, power spectral density curve

\begin{abstract}
When the components of the system begin to loose or experience other physical change, the features of the sound signal generated by the running system will be changed. One of the main contents of the noise analysis is to obtain the operating status information of equipment through the noise measurement and analysis. In this paper, the noise analysis technique is applied to the diagnosis of sensor failures. The noise signal from the sensor output is used to achieve system characteristic value, and then it is used to diagnose the operating status of the sensor. The experiment was conducted for thermowell connection failure, and the experiment result shows the efficiency of the proposed method.
\end{abstract}

\section{The background and status of noise analysis technique}

Noise is a complex sound composed of many frequency sound waves. More and more attention is paid to noise analysis in failure analysis and diagnosis, because of its convenient and non-contact measurement and information-rich carrying features. Using noise signal for fault diagnosis is an extremely rapid development of integrated applications discipline which is started from the 1960s, gradual improved in the seventies and in the eighties entered the practical stage. Spectrum analysis, narrow band spectral analysis method, the sound pressure level spectrum analysis and octave analysis methods are commonly used to analyse the noise signal.

Spectral analysis, which is a simple and practical method, has been widely used. The occurrence and development of failure can often lead to changes in the structure of the signal frequency. Spectral analysis is mainly to analyse the frequency components of the noise signal. When the device is working properly, the internal characteristic frequency of device can be calculated. When a portion of the device fails, its characteristic frequency is bound to change, therefore, diagnosis could be proceeded.

\section{Equivalent model establishment for temperature sensor}

The response time of the temperature sensor is generally composed of the response time of the internal components and the response time of the surface components. The response time of the internal components predominantly depends on the thermal conductivity $(\mathrm{k})$ of the material inside the sensor, while that of surface component depends on the films heat-transfer coefficient (h). Another factor that should be considered when engineers study process effects on response time is the ratio of internal heat-transfer resistance to the surface heat-transfer resistance. This ratio is called Biot Modulus $\left(N_{B i}\right)$ and is given by the following equation, where $r_{0}$ is the outside radius of sensor.

$$
N_{B i}=\frac{\text { internal heat transfer resistance }}{\text { surface heat transfer resistance }}=\frac{h r_{0}}{k}
$$

If the Biot Modulus is large, then the response time may change very little as $h$ is increased .However, if the Biot Modulus is small, the response time will be very sensitive to changes with h, especially in poor heat-transfer media where $\mathrm{h}$ is small. 
According to the empirical formula, the response time of the temperature sensor model is equivalent to the following formula:

$$
\tau=C_{1}+\frac{C_{2}}{h}
$$

Where $C_{1}=\frac{\rho c r_{0}^{2}}{2 k} \ln \left(r_{0} / r_{i}\right), \quad C_{2}=\frac{\rho c r_{0}}{2}, r_{i}=$ radius at which the sensing tip is located, c=specific heat capacity of the sensing portion.

\section{Development of the noise spectrum analysis based on Fourier Transform}

Since the response time of the temperature sensor is influenced by the internal and surface components, for a certain temperature sensor, in a certain temperature range, the internal components of the thermal conductivity is constant. Therefore, the obtained response time can reflect status of the surface components. In the noise spectrum analysis, Fourier transform is a key process. First, when the factory is running, progress signal is collected using a temperature sensor. Then, noise data will be extracted from the output data and then pass a low filter. Third, the power spectral density curve (PSD) of the signal is generated by using an FFT algorithm. When power spectral density curve decrease $3 \mathrm{db}$, the corresponding eigenvalues of response time can be calculated. Implementation process is shown in Figure 1.

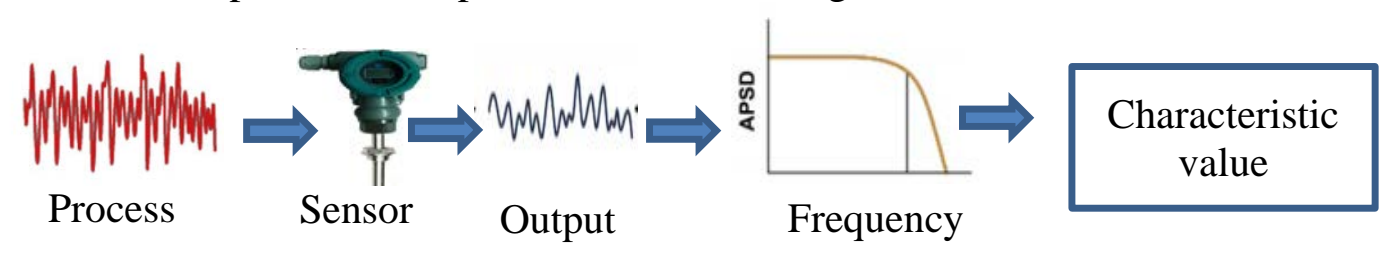

Fig.1: Realization of the noise spectrum analysis Based on Fourier Transform

Design Butterworth low-pass filter.Butterworth filter is an electronic filter, it is characterized by a maximum flat frequency response curve in the passband, no fluctuation. The frequency response curve is gradually decreased to zero in the blocking band. It can constitute four kinds filters: low pass, high pass, band pass and band-stop. After discretization, it can be used as a digital Butterworth filter. Compared with the analog filter, digital Butterworth filter has advantages of high precision, stable, flexible.

Analog low-pass filter has four technical indicators, maximum allowable passband attenuation $\left(\alpha_{p}\right)$, minimum allowable passband attenuation $\left(\alpha_{s}\right)$, the upper angular frequency of passband $\left(\Omega_{p}\right)$, and the lower limit angular frequency of stopband $\left(\Omega_{s}\right)$, respectively. There are three steps to design an analog low-pass filter based on technical indicators of the analog low-pass filter. Firstly, calculate the order of the filter. Secondly, Calculate normalized pole $\boldsymbol{P}_{\boldsymbol{k}}$ and obtain normalized transfer function $\boldsymbol{H}_{\boldsymbol{a}}(\boldsymbol{p})$.At last, remove the normalization and get the actual transfer function of the filter.

Step1: (Calculate the order of the filter) According to the definitions of maximum and minimum allowable passband attenuation as follows:

$$
\begin{gathered}
\alpha_{p}=-10 \lg \left|H_{a}\left(j \Omega_{p}\right)\right|^{2} \\
\alpha_{s}=-10 \lg \left|H_{a}\left(j \Omega_{s}\right)\right|^{2} \\
\left|H_{a}\left(j \Omega_{p}\right)\right|^{2}=\frac{1}{1+\left(\frac{\Omega}{\Omega_{c}}\right)^{2 N}}
\end{gathered}
$$

When $\Omega=\Omega_{p}$ and $\Omega=\Omega_{s},\left|H_{a}\left(j \Omega_{p}\right)\right|^{2}$ and $\left|H_{a}\left(j \Omega_{s}\right)\right|^{2}$ can be gotten in formula (5).

$$
\alpha_{\mathrm{p}}=-10 \lg \frac{1}{1+\left(\frac{\Omega_{p}}{\Omega_{c}}\right)^{2 N}}
$$




$$
\alpha_{\mathrm{p}}=-10 \lg \frac{1}{1+\left(\frac{\Omega_{S}}{\Omega_{c}}\right)^{2 N}}
$$

Then from formula (3) and formula (4), (8) and (9) can be gotten:

$$
\begin{aligned}
& 1+\left(\frac{\Omega_{p}}{\Omega_{c}}\right)^{2 N}=10^{\frac{\alpha_{\mathrm{p}}}{10}} \\
& 1+\left(\frac{\Omega_{s}}{\Omega_{c}}\right)^{2 N}=10^{\frac{\alpha_{\mathrm{s}}}{10}}
\end{aligned}
$$

Next:

$$
\left(\frac{\Omega_{p}}{\Omega_{S}}\right)^{N}=\sqrt{\frac{10^{\alpha_{p} / 10-1}}{10^{\alpha_{S} / 10-1}}}
$$

Order:

$$
\lambda_{s p}=\frac{\Omega_{s}}{\Omega_{p}}, k_{s p}=\sqrt{\frac{10^{\alpha p / 10-1}}{10^{\alpha_{s} / 10-1}}}
$$

The following formula (12) can be gotten:

$$
\mathrm{N}=-\frac{\log k_{s p}}{\log \lambda_{s p}}
$$

Step2: (Calculate normalized pole $\boldsymbol{P}_{\boldsymbol{k}}$ and obtain normalized transfer function $\boldsymbol{H}_{\boldsymbol{a}}(\boldsymbol{p})$ ) The magnitude squared function of Butterworth lowpass filter can be expressed by the following formula:

$$
\left|H_{a}(j \Omega)\right|^{2}=\frac{1}{1+\left(\frac{\Omega}{\Omega_{c}}\right)^{2 N}}
$$

Replace $\mathrm{j} \Omega$ with s, magnitude squared function can be expressed as:

$$
H_{a}(s) H_{a}(-s)=\frac{1}{1+\left(\frac{s}{\Omega_{c}}\right)^{2 N}}
$$

$\mathrm{s}=\sigma+\mathrm{j} \Omega$, this formula shows that there are $2 \mathrm{~N}$ poles in magnitude squared function . Pole $s_{k}$ can be expressed by the following formula:

$$
s_{k}=\left(j \Omega_{c} e^{\left.\frac{j \pi(2 k+1)}{2 N}\right)}=\Omega_{c} e^{\frac{\pi}{2}} e^{j \pi \frac{2 k+1}{2 N}}=\Omega_{c} e^{j \pi\left(\frac{1}{2}+\frac{2 k+1}{2 N}\right)}\right.
$$

$\mathrm{k}=0,1,2, \ldots, 2 \mathrm{~N}-1$. As shown in Figure 2, the poles equally spaced in a circle which radius is $\Omega_{c}$, each interval between two poles is $\pi / \mathrm{N}$. 


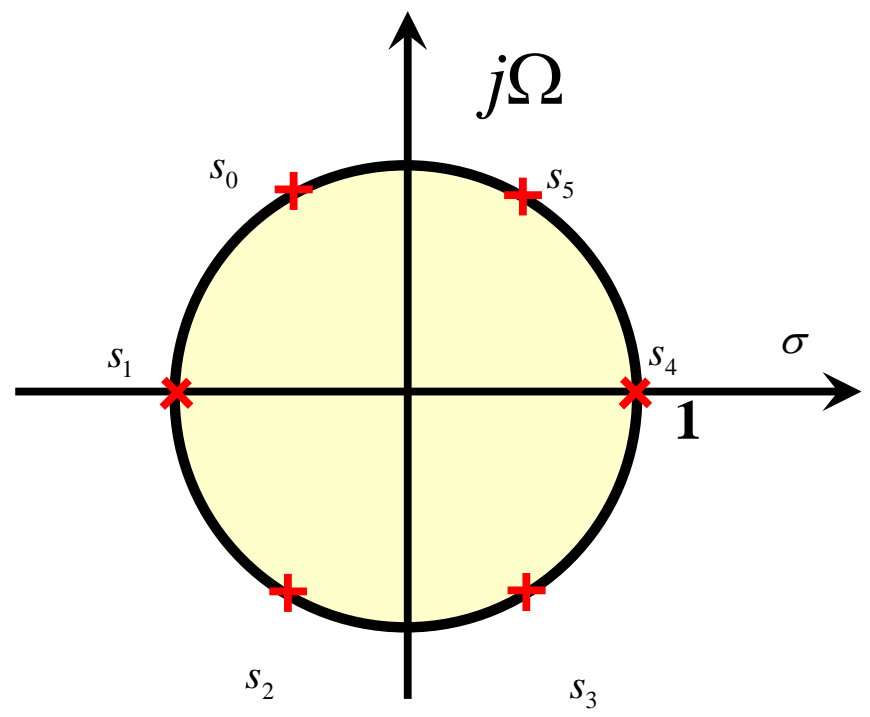

Fig.2: Distribution of third-order Butterworth filter pole

In order to form a stable filter, $H_{a}(s)$ is constituted by the left half plane poles, while $H_{a}(-s)$ is constituted by the right half plane poles. Expression of $H_{a}(s)$ is shown as below.

$$
H_{a}(s)=\frac{\Omega_{c}^{N}}{\prod_{k=0}^{N-1}\left(s-s_{k}\right)}
$$

For example, if $\mathrm{N}=3$, six poles are calculated as follows:

$$
s_{0}=\Omega_{c} e^{j \frac{2}{3} \pi}, s_{1}=\Omega_{c} e^{j \pi}, s_{2}=\Omega_{c} e^{j \frac{4}{3} \pi}, s_{3}=\Omega_{c} e^{j \frac{5}{3} \pi}, s_{4}=\Omega_{c} e^{j 2 \pi}, s_{5}=\Omega_{c} e^{j \frac{7}{3} \pi}
$$

Three poles in the left half plane are shown as following respectively.

$$
s_{0}=\Omega_{c} e^{j \frac{2}{3} \pi}, s_{1}=\Omega_{c} e^{j \pi}, s_{2}=\Omega_{c} e^{j \frac{4}{3} \pi}
$$

Next, $H_{a}(s)$ is be given by the following formula

$$
H_{a}(s)=\frac{\Omega_{c}{ }^{3}}{\left(s-\Omega_{c} e^{j \frac{2}{3} \pi}\right)\left(s-\Omega_{c} e^{j \pi}\right)\left(s-\Omega_{c} e^{j \frac{4}{3} \pi}\right)}
$$

Order $\mathrm{p}=\frac{s}{\Omega_{c}}=\frac{j \Omega}{\Omega_{c}}=j \lambda, \mathrm{p}$ is a normalized Laplace complex variable. $\lambda=\Omega / \Omega_{c}$ and $\lambda$ is normalized frequency. After normalization, the transfer function of Butterworth filter can be given in the following formula.

$$
\mathrm{H}_{a}(p)=\frac{1}{\prod_{K=0}^{N-1}\left(p-p_{k}\right)}
$$

In the formula, $p_{k}={ }^{s_{k}} / \Omega_{c}$ and $p_{k}$ is normalized pole. $s_{k}$ can be represented by the left half plane pole.

$$
p_{k}=e^{j \pi\left(\frac{1}{2}+\frac{2 k+1}{2 N}\right)}, k=0,1, \ldots N-1
$$

Then $\mathrm{H}_{a}(p)$ can be given as followed

$$
\mathrm{H}_{a}(p)=\frac{1}{a_{0}+a_{1} p+a_{2} p^{2}+\cdots+a_{N-1} p^{N-1}+p^{N}}
$$




\section{Step3: (Remove the normalization and get the actual transfer function of the filter)}

Order $\boldsymbol{p}=\frac{\boldsymbol{s}}{\boldsymbol{\Omega}_{\boldsymbol{c}}}$ in $\boldsymbol{H}_{\boldsymbol{a}}(\boldsymbol{p})$, we can get the actual transfer function $\left(\boldsymbol{H}_{\boldsymbol{a}}(\boldsymbol{s})\right)$ of the filter.

The implementation of welch power spectral estimation method.Power spectral estimation plays a key role in signal analysis and recognition, which is widely used in radar, sonar, speech, seismology and biomedicine. Figure period spectral estimation is a widely used method, because that it has a lot of character such as clear physical concept, simple method and high efficiency. However, figure cycle method cannot meet requirements of consistency estimate. The contradiction between resolution and variance performance makes the algorithm difficult to acquire good performance and that cannot meet the actual needs of the signal spectrum estimation. In this regard, a lot of improvement algorithms have been proposed depending on the application and welch algorithm is one of them. Through data segmentation and adding windows, this algorithm can effectively reduce the spectral estimation variance without introduce severe damage. These features make the welch become an effective method of spectral estimation.

In order to obtain power spectral density, the implementation of welch method can be divided into the following three steps.

Firstly, divide $x_{n}(n)$ into L sections. Welch method allows each section of data has overlapping parts. If there is half overlapping in each section, then the number of segments will be as follows.

$$
\mathrm{L}=\frac{N-M / 2}{M / 2}
$$

Second, in order to divide a long sequence into a short sequence in finite length, the spectrum distortion will occur and that will introduce spectral energy leakage. , A window function is always used to reduce this spectrum energy leakage. There are some commonly used window functions, for example, Rectangular window, Bartlett window, Hanning window, Hamming window and Blackman window. In welch method, the data window is not limited to a rectangular window, and Hanning window, Hamming window or other window can also be used. Record this data window as $d_{2}(n)$. Applying Fourier transform in the each small windowed piece and the following formula can be given:

$$
P_{P e r}^{i}(w)=\frac{1}{M U}\left|\sum_{n=0}^{M-1} x_{N}^{i}(n) d_{2}(n) e^{-j w n}\right|^{2}
$$

Where:

$$
\mathrm{U}=\frac{1}{M} \sum_{n=0}^{M-1} d_{2}^{2}(n)
$$

$\mathrm{U}$ is a normalization factor. It is used to ensure that the spectrum result is gradual unbiased estimator

Third, strike the average value of each subsection.

$$
P_{P E R}(\mathrm{w})=\frac{1}{L} \sum_{i=1}^{L} P_{P E R}^{i}(w)=\frac{1}{M U L} \sum_{i=1}^{L}\left|\sum_{n=0}^{M-1} x_{N}^{i}(n) d_{2}(n) e^{-j w n}\right|^{2}
$$

\section{Acquisition and processing of experimental data}

Temperature sensor thermowell is used to validate noise analysis approach for the failure modes of loose connection. In order to simulate the status of the loose connection of thermowell, a temperature sensor without thermowell is used. In different cases that the sensors have a thermowell or not, they are put into boiling water. Then, the processing results from temperature transmitter will be sent to a computer and the data will be shown.

In the case that the sensor element has a thermowell, this will increase the surface heat transfer resistance. Based on Biot modulus formula (1), this will reduce the value of film heat-transfer coefficient h. what's more, according to the Biot modulus formula (2), the decrease of heat transfer coefficient will lead to the increase of response time. As is shown in Figure 3, when the sensor 
element without thermowell, the power spectral density curve obtained is represented by a dotted line and when the sensing element with a thermowell, the power spectral density curve obtained is indicated by a solid line in the figure. When the value of power spectral density decreases for 3db, the response frequency is represented by $\mathrm{f} 1 \mathrm{f} 2$, respectively. In the figure, $\mathrm{t} 1>\mathrm{t} 2$ as $\mathrm{f} 2>\mathrm{f} 1 \mathrm{can}$ be gotten according to the formula $t=1 / f$. In other words, the response time of the sensing element with a thermowell should be greater than the sensing element without thermowell.

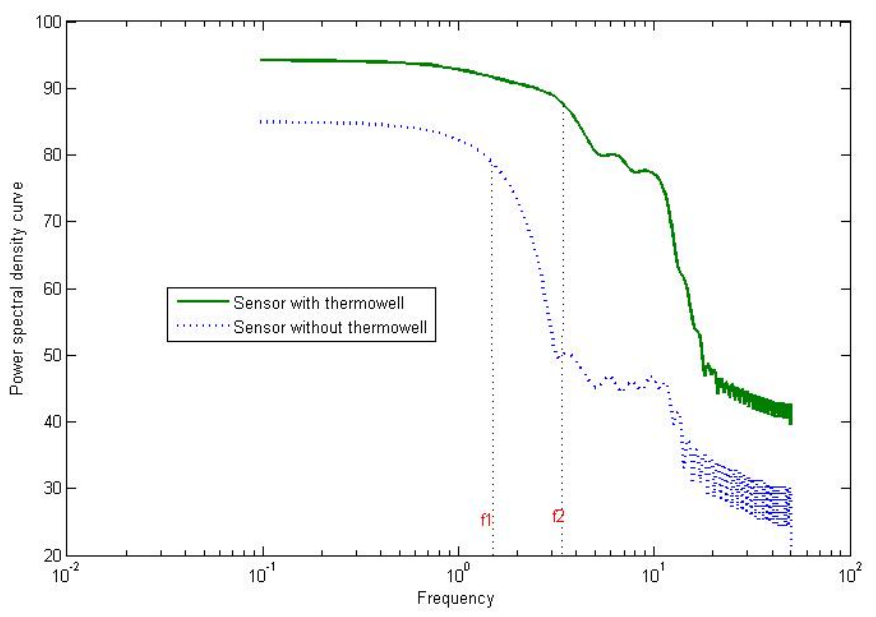

Fig.3: Experimental results

\section{Conclusion}

In this paper, the noise analysis technique is applied to diagnose sensor failures, the implementation process of noise analysis is described in a very detailed way. Thermowell loose connection failure temperature sensor is selected to this validate noise analysing method. Through the process of collecting data, lowpass filtering, and striking power spectral density and calculating system response time, the validation shows the feasibility of this noise analysis approach which can be applied in the sensor failure diagnosis for industrial transmitter.

\section{Acknowledgements}

The research work was supported by the National High Technology Development Plan (863):Largescale Architecture of PLC under contract 2013AA040301.

\section{References}

[1] Hashemian H M. On-line monitoring applications in nuclear power plants [J]. Progress in Nuclear Energy, 2011, 53(2): 167-181

[2] Hashemian H M. Maintenance of process instrumentation in nuclear power plants [M]. Springer, 2006.

[3] Lu Chen. main bearing condition monitoring and fault diagnosis based on noise analysis: [dissertation] Dalian: Dalian University of Technology [J] 2002

[4] Gao Ning, Huang Yulong. fault analysis system for noise signals [J] industrial control computer, 2006, 19 (1): 14-15.

[5] Zheng Keyuan. Design and implementation of noise analysis FFT algorithm [D] Xi'an University of Electronic Science and Technology, 2013. 